Journal of Applied AnALysis

Vol. 11, No. 2 (2005), pp. 187-205

\title{
PROJECTIONS IN WEAKLY COMPACTLY GENERATED BANACH SPACES AND CHANG'S CONJECTURE
}

\author{
P. KOSZMIDER
}

Received May 28, 2003 and, in revised form, September 26, 2004

\begin{abstract}
Classical results on weakly compactly generated (WCG) Banach spaces imply the existence of projectional resolutions of identity (PRI) and the existence of many projections on separable subspaces (SCP). We address the questions if these can be the only projections in a nonseparable WCG space, in the sense that there is a PRI $\left(P_{\alpha}: \omega \leq \alpha \leq \lambda\right)$ such that any projection is the sum of an operator in the closure of the linear span of countably many $P_{\alpha}$ 's (in the strong operator topology) and a separable range operator. Wark's modification of Shelah's and Steprāns' construction provides an unconditional example for $\lambda=\omega_{1}$. We note that it is impossible for $\lambda>\omega_{2}$.
\end{abstract}

2000 Mathematics Subject Classification. Primary 46B26, 03E35.

Key words and phrases. Weakly compactly generated Banach spaces, Chang's conjecture, few operators.

The author would like to thank Prof. S. Todorčević for explaining that the earlier versions of this paper (from March 2000) should be rewritten using the notion of a WCG Banach space rather than previous quite technical condition. This part of the research was supported by Université Paris VII and by Research Foundation of the State of São Paulo (FAPESP Proc No. 01/03820-1).

ISSN 1425-6908 C Heldermann Verlag. 


\begin{abstract}
. The main result of the paper is that for $\lambda=\omega_{2}$, the second uncountable cardinal, the question is logically undecidable and depends on additional axioms deciding the combinatorics on $\omega_{2}$; for example Chang's conjecture implies that there are other projections than the projections mentioned above. The full strength results concern all linear operators not just the projections.
\end{abstract}

\title{
1. Introduction
}

Let $X$ be a weakly compactly generated (i.e., equal to the closure of the linear span of a subset which is compact in the weak topology and abbreviated WCG in the sequel) Banach space of density $\lambda$, where $\lambda$ is an infinite cardinal. Amir and Lindenstrauss [1] (see also [5]) showed that in any WCG Banach space $X$ there is a sequence of projections $\left(P_{\alpha}: \omega \leq \alpha \leq\right.$ $\lambda$ ) called a projectional resolution of identity which satisfies for $\omega \leq \alpha \leq \lambda$ :

i) $\left\|P_{\alpha}\right\|=1$,

ii) $P_{\alpha} \circ P_{\beta}=P_{\beta} \circ P_{\alpha}=P_{\alpha}$ whenever $\omega \leq \alpha \leq \beta \leq \lambda$,

iii) the density of the range of $P_{\alpha}$ is not bigger than the cardinality of $\alpha$,

iv) $\bigcup\left\{P_{\beta}[X]: \beta<\alpha\right\}$ is norm dense in $P_{\alpha}[X]$ if $\alpha$ is a limit ordinal,

v) $P_{\lambda}=I d_{X}$.

As a closed subspace $Y$ is a range of a projection in a Banach space $X$ if and only if $X=Y \oplus Z$ for some closed subspace $Z$, i.e., if and only if $Y$ is complemented in $X$, the projectional resolution of identity provides a continuous, increasing, well-ordered sequence of small complemented subspaces whose union is the entire space.

Amir and Lindenstrauss also proved that for any separable subspace $Y$ of $X$ as above there is a range of a projection (i.e., a complemented subspace) which is separable and includes $Y$. It is natural to ask if these projections can generate, in a sense, all projections in $X$.

In [17] Shelah and Steprāns constructed a Banach space of density $\omega_{1}$ where any operator is a multiplication by a constant plus a separable range operator. In [21] Wark modified it to obtain a WCG space with these properties. Of course such a space has the property that for any PRI, $\left(P_{\alpha}: \omega \leq \alpha \leq \omega_{1}\right)$ any projection is the sum of a multiple of $P_{\omega_{1}}$, and a separable range projection. In [2] Argyros, Lopez-Abad and Todorčević were able to eliminate many operators with separable ranges from such spaces. Things complicate if we consider spaces of bigger densities $\lambda>\omega_{1}$, as $P_{\omega_{1}}$ is an operator which is not a multiple of the identity plus a separable range operator.

Definition 1.1. We say that a weakly compactly generated Banach space $X$ has few operators if and only if there is a PRI, $\left(P_{\alpha}: \omega \leq \alpha \leq \lambda\right)$ such 
that any operator $T: X \rightarrow X$ is of the form $P+S$ where $P$ is in the closure of the linear span of countably many $P_{\alpha}$ 's (in the strong operator topology) and $S$ has a separable range.

In Section 2 we follow Wark's modification of Shelah's and Steprāns' construction and obtain a consistent example of a WCG Banach space of density $\omega_{2}$ which has few operators. The new feature is the use of a family of countable subsets of $\omega_{2}$ which plays the role of the initial fragments of $\omega_{1}$ to define the norm. Such family is added in Section 3 by forcing (see [11]), exists only consistently and can be considered as a version of a simplified morass (see [20]).

In Section 4 we assume a model-theoretic statement known as Chang's Conjecture which implies the non-existence of the families of Section 2. Under this assumption we prove that for any WCG Banach space $X$ of density $\lambda=\omega_{2}$ for any PRI there is a projection which witnesses the fact that $X$ has more than few operators. Thus the question of the existence of WCG Banach space of density $\omega_{2}$ with few operators is undecidable based on the usual axioms of set theory. Section 4 is concluded with remarks on diagonal operators, some questions and the observation that there are no WCG Banach spaces of density bigger than $\omega_{2}$ with few operators.

From the point of view of infinitary combinatorics we deal with a pair of two cardinal invariants $(\kappa, \lambda)$ on WCG Banach spaces, the density $\lambda$ and $\kappa$ such that any operator is "nice" plus an operator with the range of density $\kappa$. However the paper [17] does not mention it, the constructions for the pairs of the form $\left(\kappa, \kappa^{+}\right)$can be done in a similar way. (The paper [16] mentions it, but in the context of some stronger assumption. There is still some gray area concerning the assumptions needed for these constructions.) The modification as in [21] can be done as well. In this paper we are interested in the first pair of the form $\left(\kappa, \kappa^{++}\right)$that is $\left(\omega, \omega_{2}\right)$ and later in the pairs $(\omega, \lambda)$ for $\lambda>\omega_{2}$. This growth of the gap between cardinal invariants of the structures and the logical and combinatorial phenomena which accompany it, is a classical theme in infinitary combinatorics and the results we obtain follow the general pattern for mathematical structures other than Banach spaces (see for example [3], [9], [10], [18], [19], [22]). It is related to Ramsey theoretic properties of the infinite cardinals.

The notation concerning infinitary combinatorics and forcing follows [11], in particular $\kappa, \lambda$ denote cardinals $[X]^{\kappa}$ denotes subsets of $X$ of cardinality $\kappa$, $[X]^{<\omega}$ denotes finite subsets of $X$. By models we mean models of fragments of ZFC. Basics on the use of elementary submodels outside logic can be found in $[6]$.

The notation and terminology concerning Banach spaces follows for example [4] in particular $[Y]$ denotes the norm closure of the linear space generated by $Y$ in a Banach space; $X^{*}$ denotes the dual space; $\left\{\left(x_{\alpha}, f_{\alpha}\right): \alpha<\kappa\right\}$ 
is called a Marcuševič's basis if $f_{\alpha}\left(x_{\beta}\right)=1$ if $\alpha=\beta$ and $f_{\alpha}\left(x_{\beta}\right)=0$ if $\alpha \neq \beta$, $\left[\left\{x_{\alpha}: \alpha<\kappa\right\}\right]=X$ and $f_{\alpha}(x)=0$ for all $\alpha \in \kappa$ implies that $x=0$; strong topology on the space of operators is the one of pointwise convergence (see $[7])$.

\section{The construction of the space}

Let $\lambda$ be an uncountable cardinal. In this section we follow the paper [17] and its modification [21], some objects related to $\omega_{1}$ in [17] are appropriately changed to objects related to some family in $[\lambda]^{\omega}$ whose existence follow, for $\lambda=\omega_{2}$, from strong negations of Chang's Conjecture, which are discussed in the following section. So, we assume the existence of families $\mathcal{A}, \mathcal{B}, \mathcal{D}$ and $\mathcal{F}$; the families $\mathcal{A}, \mathcal{B}, \mathcal{D}$ correspond directly to the families from [17] and the family $\mathcal{F}$ plays the role of the initial fragments of $\omega_{1}$. We will require the following properties:

A1) $\mathcal{A} \subseteq[\lambda]^{<\omega}$.

A2) $\bigcup \mathcal{A}=\lambda$.

A3) $\mathcal{A}$ is closed under subsets.

B1) $\mathcal{B} \subseteq[\lambda]^{<\omega}$.

B2) $\mathcal{B}$ is closed under subsets.

$\mathrm{AB} 1) \mathcal{A} \cap \mathcal{B} \subseteq[\lambda]^{1}$.

AB2) For every family $\left\{a_{\xi}: \xi<\omega_{1}\right\} \subseteq[\lambda]^{2}$ of disjoint pairs and $k \in \omega$ there are $\xi_{1}<\xi_{2}<\ldots<\xi_{k}<\omega_{1}$ such that

$$
\begin{array}{r}
\left\{a_{\xi_{1}}(1), \ldots, a_{\xi_{k}}(1)\right\} \in \mathcal{A}, \\
\left\{a_{\xi_{1}}(0), \ldots, a_{\xi_{k}}(0)\right\} \in \mathcal{B}
\end{array}
$$

where $a_{\xi}=\left\{a_{\xi}(0), a_{\xi}(1)\right\}$ for $\xi \in \omega_{1}$.

D1) $\mathcal{D} \subseteq\left[[\lambda]^{2}\right]^{<\omega}$ and for every $a, b \in D \in \mathcal{D}$ we have either $\max (a)<$ $\min (b)(a<b)$ or $\max (b)<\min (a)(b<a)$; we express this property by saying that elements of $\mathcal{D}$ consist of consecutive pairs.

D2) If $D, D^{\prime} \in \mathcal{D}$ are distinct, then there may be at most five pairs in $D$ which intersect other than itself pair from $D^{\prime}$ i.e.,

$$
\left|\left\{a \in D: a \cap\left(\bigcup\left(D^{\prime}-\{a\}\right)\right) \neq \emptyset\right\}\right| \leq 5 .
$$

D3) Whenever $\left\{d_{\xi}: \xi<\omega_{1}\right\} \subseteq[\lambda]^{2}$ is a collection of consecutive pairs and $k \in \omega$, then there are $\xi_{1}<\xi_{2}, \ldots,<\xi_{k}<\omega_{1}$ such that $\left\{d_{\xi_{i}}: 1 \leq i \leq\right.$ $k\} \in \mathcal{D}$.

D4) Whenever $D \in \mathcal{D}$ and $\alpha<\lambda$, and $X \subseteq \lambda-\alpha$ is countable, there is $D^{\prime} \in \mathcal{D}$ such that

$\left(\bigcup D^{\prime}\right) \cap X=\emptyset, \quad D \cap[\alpha]^{2}=D^{\prime} \cap[\alpha]^{2}, \quad(\bigcup D) \cap \alpha=\left(\bigcup D^{\prime}\right) \cap \alpha$.

AD1) Whenever $a \in \mathcal{A}$ and $D \in \mathcal{D}$, then $|a \cap(\bigcup D)| \leq 2$. 
BD1) Whenever $a \in \mathcal{B}$ and $D \in \mathcal{D}$, then $|a \cap(\bigcup D)| \leq 2$.

F1) $\mathcal{F} \subseteq[\lambda]^{\omega}$ is cofinal in $[\lambda]^{\omega}$.

DF1) Suppose that $D \in \mathcal{D}, d, d^{\prime}, d^{\prime \prime} \in D$ and $d<d^{\prime}<d^{\prime \prime}$ and moreover that $X \in \mathcal{F}$ is such that $d^{\prime} \cap X$ and $d^{\prime \prime} \cap X$ are both nonempty. Then $d \subseteq X$.

This completes the list of properties of the families. The reader might have noted that if not for D3), the families $\mathcal{D}=\emptyset$ and $\mathcal{F}=[\lambda]^{\omega}$ work. This is exploited in the proof of Theorem 1.9.

Now, let us define our Banach space $B$. We start with the set ${ }^{\lambda} \mathbb{R}$, that is, all functions from $\lambda$ into the reals. Following [17] and [21], we define

$$
\|f\|_{\mathcal{A}}=\sup \left\{\sqrt{\Sigma\left\{f(\alpha)^{2}: \alpha \in a\right\}}: a \in \mathcal{A}\right\}
$$

and

$$
\left.\nu_{\mathcal{D}}(f)=\sup \left\{\sqrt{\Sigma\left\{[f(\alpha)-f(\beta)]^{2}\right.}:\{\alpha, \beta\} \in D\right\}: D \in \mathcal{D}\right\} .
$$

We put $B_{*}(\mathcal{A}, \mathcal{D})=\left\{f \in^{\lambda} \mathbb{R}: \nu_{\mathcal{D}}(f)+\|f\|_{\mathcal{A}}\right.$ is finite $\}$. Using A1), A2) and D1) one can calculate that $\left(B_{*}(\mathcal{A}, \mathcal{D}), \nu_{\mathcal{D}}+\|\|_{\mathcal{A}}\right)$ are Banach spaces. Namely they are clearly linear spaces and the usual triangle inequality for $l_{2}(\lambda)$ implies that they are normed spaces. Given a Cauchy sequence, one gets its uniform coordinate-wise limit by the completness of $l_{\infty}(\lambda)$, it has to belong to the spaces since $\nu_{\mathcal{D}}+\|\|_{\mathcal{A}}$ can be approximated on finite sets using terms of the sequence which must be norm-bounded.

For every $X \subseteq \lambda$, by $1_{X}$ we denote the characteristic function of $X$. By $\phi_{\{\alpha\}}$ we define the functional satisfying $\phi(f)=f(\alpha)$ for $\alpha \in \lambda$. For every $X \subseteq \lambda$ we define $B_{X}(\mathcal{A}, \mathcal{D})$ to be the closure of the linear span $\left\{1_{\{\alpha\}}: \alpha \in\right.$ $X\}$ in $B_{*}(\mathcal{A}, \mathcal{D})$ with respect to the norm $\nu_{\mathcal{D}}+\|\|_{\mathcal{A}}$. The main result will concern the space $\left(B_{\lambda}(\mathcal{A}, \mathcal{D}), \nu_{\mathcal{D}}+\|\|_{\mathcal{A}}\right)$, however we will consider the space $B_{X}(\mathcal{A}, \mathcal{D})$ for $X \in \mathcal{F}$ as in F1) and for $X$ an ordinal less than $\lambda$, in the latter case we will call this subspace an initial block. The projection on the initial block $\alpha$ means the restriction of a function to $\alpha$.

Lemma 2.1. Suppose $\mathcal{A}$ and $\mathcal{B}$ are such families that there exist families $\mathcal{D}$ and $\mathcal{F}$ such that the properties $\mathrm{A} 1)-\mathrm{DF} 1)$ with the possible exception of the property $\mathrm{D} 3)$ are satisfied, then the space $\left(B_{\lambda}(\mathcal{A}, \mathcal{D}), \nu_{\mathcal{D}}+\|\|_{\mathcal{A}}\right)$ has the following properties:

1) The sequence $\left\{\left(1_{\{\alpha\}}, \phi_{\{\alpha\}}\right): \alpha<\lambda\right\}$ is a Marcuševič basis for the space.

2) The space is weakly compactly generated and $\left(P_{\alpha}: \omega \leq \alpha \leq \lambda\right)$ is a PRI for $B_{\lambda}(\mathcal{A}, \mathcal{D})$ where $P_{\alpha}$ is the projection on the initial block $\alpha$.

3) Every operator defined on the space is of the sum of a separable range operator and a diagonal operator with respect to the basis $\left\{1_{\{\alpha\}}: \alpha<\lambda\right\}$.

4) If additionally $\mathcal{D}$ satisfies D3) then every operator on the space is the sum of a separable range operator and an operator in the closure (in 
the strong operator topology) of the space spanned by a countable set of the projections $P_{\alpha}$ for $\omega \leq \alpha \leq \lambda$.

Proof. Let us fix the families satisfying the properties A1)-DF2) with the possible exception of D3). Let us introduce the notation $B_{\xi}=B_{\xi}(\mathcal{A}, \mathcal{D})$, $B=B_{\lambda}, \nu=\nu_{\mathcal{D}}$ and \|\|$=\nu+\|\|_{\mathcal{A}}$. So we are working with the space $(B,\|\|)$. It is clear that the sequence $\left\{\left(1_{\{\alpha\}}, \phi_{\{\alpha\}}\right): \alpha<\lambda\right\}$ is a Marcuševič's basis i.e., 1) holds.

Note that if $f \in l_{2}(\lambda)$, then $f \in B$ i.e., $T: l_{2}(\lambda) \rightarrow B$ defined as the formal identity on $l_{2}(\lambda)$ is a well-defined injection. It is clear that it is linear and $\nu(f)+\|f\|_{\mathcal{A}} \leq 3\left(\|f\|_{l_{2}(\lambda)}\right)$, that is, $T$ is a bounded operator. All vectors $1_{\{\alpha\}}$ belong to $l_{2}(\lambda)$, i.e., the range of $T$ is dense in $B$.

Now, by Banach-Alaoglu theorem the balls in the reflexive space $l_{2}(\lambda)$ are weakly compact, and hence, their images under $T$ are weakly compact as well, but their union is dense in $B$, i.e., $B$ is weakly compactly generated. To complete the proof of 2 ) one needs to prove the following:

Claim 1. $\left\|P_{\alpha}\right\| \leq 1$.

Proof of the Claim 1. It is clear that $\left\|P_{\alpha}(f)\right\|_{\mathcal{A}} \leq\|f\|_{\mathcal{A}}$. So we need to prove that $\nu_{\mathcal{D}}\left(P_{\alpha}(f)\right) \leq \nu_{\mathcal{D}}(f)$ for every $f \in B_{\lambda}$. Note that if $f \in B_{\lambda}$, then $\{\beta \in \lambda: f(\beta) \neq 0\}$ is at most countable. This follows from the fact that the span of $\left\{1_{\{\beta\}}: \beta \in \lambda\right\}$ is norm dense in $B_{\lambda}$ and all singletons belong to $\mathcal{A}$ (which follows from A2) ed A3)).

So, given $D \in \mathcal{D}$ consider $D^{\prime} \in \mathcal{D}$ as in D4) for $X=\{\beta: f(\beta) \neq 0\}-\alpha$. It is enough to note that

$$
\begin{aligned}
& \sqrt{\Sigma\left\{\left(P_{\alpha}(f(\beta))-P_{\alpha}\left(f\left(\beta^{\prime}\right)\right)\right)^{2}:\left\{\beta, \beta^{\prime}\right\} \in D\right\}} \\
& =\sqrt{\Sigma\left\{\left(f(\beta)-f\left(\beta^{\prime}\right)\right)^{2}:\left\{\beta, \beta^{\prime}\right\} \in D^{\prime}\right\}} .
\end{aligned}
$$

But this follows directly from D4) which implies that the numbers appearing in the above expressions are equal respectively.

Claim 2. If $X \in \mathcal{F}$ then $\pi_{X}: B \rightarrow B_{X}$ given by $\pi_{X}(f)=f \mid X$ is a bounded operator.

Proof of the Claim 2. It will be enough to see that we have

$$
\|y\| \leq 3\|y+z\|
$$

for every $y \in B_{X}, z \in B_{\lambda-X}$. It is easy to see (using A2)) that for $y, z$ as above, we have $y(\alpha)=(y+z)(\alpha)$ for $\alpha \in X$ and $z(\alpha)=(y+z)(\alpha)$ for $\alpha \in \lambda-X$. This implies that $\|y\|_{\mathcal{A}} \leq\|y+z\|_{\mathcal{A}}$. We also have

$$
\begin{aligned}
\nu(y) & =\sup \left\{\Sigma\left\{\sqrt{[y(\alpha)-y(\beta)]^{2}}:\{\alpha, \beta\} \in D\right\}: D \in \mathcal{D}\right\} \\
& \leq \sup \left\{\Sigma\left\{\sqrt{[(y+z)(\alpha)-(y+z)(\beta)]^{2}}:\{\alpha, \beta\} \in D\right\}: D \in \mathcal{D}\right\}
\end{aligned}
$$




$$
\left.+2 \sup \left\{\sqrt{y(\alpha)^{2}}:\{\alpha, \beta\} \in D\right\}: \alpha<\xi<\beta, D \in \mathcal{D}\right\} \leq \nu(y+z)+2\|y\|_{\mathcal{A}} .
$$

Here we used the fact that there are at most two pairs in $D \in \mathcal{D}$ which are separated by $X$ (i.e., one element of the pair belongs to $X$ and the other does not) which follows from DF1), also A2) is used for the second term. So we get

$$
\|y\| \leq \nu(y+z)+2\|y\|_{\mathcal{A}}+\|y\|_{\mathcal{A}} \leq \nu(y+z)+3\|y+z\|_{\mathcal{A}} \leq 3(\|y+z\|) .
$$

This completes the proof of the claim.

Following the organization of the paper [17] we state now the following

Claim 3. There is a constant $M$ such that $\nu\left(1_{b}\right) \leq M$ for all $b \in \mathcal{B}$.

Proof of the Claim 3. It follows directly from BD1).

Now we are ready to make a similar argument to that of Lemma 1 of [17]. Suppose that $T: B \rightarrow B$ is a bounded operator which is not of the desired (by 3$)$ ) form. Then there is a family $\left\{a_{\xi}: \xi<\omega_{1}\right\}$ of disjoint pairs of $\lambda$ such that $T\left(1_{\left\{a_{\xi}(0)\right\}}\right)\left(a_{\xi}(1)\right) \neq 0$. To see why this is so, note that otherwise there is a countable $Y \subseteq \lambda$ such that whenever $\alpha \in \lambda-Y$, then $T\left(1_{\{\alpha\}}\right)=r_{\alpha} 1_{\{\alpha\}}+f_{\alpha}$ where $f_{\alpha} \in B_{Y}$. Since $\mathcal{F}$ is a cofinal family in $[\lambda]^{\omega}$, we may w.l.o.g. assume that $Y=X \in \mathcal{F}$. This implies that

$$
\begin{aligned}
T(x) & =T\left(\pi_{X}(x)+\pi_{\lambda-X}(x)\right) T\left(\pi_{X}(x)\right)+T\left(\pi_{\lambda-X}(x)\right) \\
& =T\left(\pi_{X}(x)\right)+\pi_{X}\left(T\left(\pi_{\lambda-X}(x)\right)\right)+\pi_{\lambda-X}\left(T\left(\pi_{\lambda-X}(x)\right)\right) \\
& =S_{1}+S_{2}+D
\end{aligned}
$$

where $D$ is diagonal and $S_{1}, S_{2}$ have separable ranges. The above operators are also bounded by Claim 2 .

Notice that the family of pairs may be thinned out so that

$$
T\left(1_{\left\{a_{\xi}(0)\right\}}\right)\left(a_{\eta}(1)\right)=0
$$

for $\xi \neq \eta$. The reason is that otherwise there is an uncountable set $Y$, positive $\delta$ and $\eta_{0} \in \omega_{1}$ such that

$$
\left|T\left(1_{\left\{a_{\xi}(0)\right\}}\right)\left(a_{\left\{\eta_{0}(1)\right\}}\right)\right|>\delta
$$

for all $\xi \in Y$ and all the numbers $T\left(1_{\left\{a_{\xi}(0)\right\}}\right)\left(a_{\left\{\eta_{0}(1)\right\}}\right)$ have the same sign. Let $n \delta \geq(M+1)\|T\|$. An application of AB2) yields $X \in[Y]^{n}$ such that $\left\{a_{\xi}(0): \xi \in X\right\} \in \mathcal{B}$ and hence $\nu\left(1_{\left\{a_{\xi}(0): \xi \in X\right\}}\right) \leq M$ by Claim 3 , and $\|\left(1_{\left\{a_{\xi}(0): \xi \in X\right\}} \|_{\mathcal{A}} \leq 1\right.$ by AB1), thus $\|\left(1_{\left\{a_{\xi}(0): \xi \in X\right\}} \| \leq 1+M\right.$. On the other hand

$$
\left\|T\left(1_{\left\{a_{\xi}(0): \xi \in X\right\}}\right)\right\| \geq\left\|T\left(1_{\left\{a_{\xi}(0): \xi \in X\right\}}\right)\right\|_{\mathcal{A}}>n \delta \geq(M+1)\|T\|,
$$

a contradiction. 
It may also be assumed that there is $\rho>0$ such that $\left|T\left(1_{\left\{a_{\xi}(0)\right\}}\right)\left(a_{\xi}(1)\right)\right|>$ $\rho$ for $\xi \in \omega_{1}$ and all the numbers $T\left(1_{\left\{a_{\xi}(0)\right\}}\right)\left(a_{\xi}(1)\right)$ have the same sign. Choose $k \in \omega$ such that $\sqrt{k} \rho \geq(M+1)\|T\|$. Now use AB2) to find $X \in\left[\omega_{1}\right]^{k}$ such that $\left\{a_{\xi}(0): \xi \in X\right\} \in \mathcal{B}$ and $\left\{a_{\xi}(1): \xi \in\right.$ $X\} \in \mathcal{A}$. It follows from Claim 3 that $\nu\left(1_{\left\{a_{\xi}(0): \xi \in X\right\}}\right) \leq M$ and then from AB1) that $\|\left(1_{\left\{a_{\xi}(0): \xi \in X\right\}} \| \leq 1+M\right.$. While $(*)$ above gives that $\left|T\left(1_{\left\{a_{\xi}(0): \xi \in X\right\}}\right)\left(a_{\xi^{\prime}(1)}\right)\right|>\rho$ for any $\xi^{\prime} \in X$, so

$$
\left\|T\left(1_{\left\{a_{\xi}(0): \xi \in X\right\}}\right)\right\| \geq\left\|T\left(1_{\left\{a_{\xi}(0): \xi \in X\right\}}\right)\right\|_{\mathcal{A}}>\sqrt{k} \rho \geq(M+1)\|T\|,
$$

which is a contradiction and completes the proof of 3 ).

To prove 4) we will assume that $T$ is a diagonal operator, which can be done by 3 ). First, note the following

Claim 4. If $D \in \mathcal{D}$, then $\nu\left(1_{\cup D}\right) \leq \sqrt{5}$.

Proof of the Claim 4. It follows directly from D2).

We will also need the following

Claim 5. Suppose that $T: B \rightarrow B$ is a diagonal, bounded operator satisfying for $\alpha<\lambda$

$$
T\left(1_{\{\alpha\}}\right)=\rho_{\alpha} 1_{\{\alpha\}} .
$$

Then, if $T$ is not in the closure (in the strong operator topology) of the linear span of countably many projections on initial blocks, then there are uncountably many consecutive pairs $\left\{a_{\xi}: \xi<\omega_{1}\right\}$ such that

$$
\left|\rho_{\alpha_{\xi}(0)}-\rho_{\alpha_{\xi}(1)}\right|>0
$$

where $a_{\xi}(0)<a_{\xi}(1)$.

Proof of the Claim 5. Consider maximal, with respect to inclusion, ordinal intervals $[\alpha, \beta)$ such that $\rho_{\alpha}=\rho_{\gamma}$ for all $\alpha \leq \gamma<\beta$. Clearly they are disjoint when different and they cover $\lambda$. If there are uncountably many of them one can choose a sequence of them $\left(\left[\alpha_{\xi}(0), \alpha_{\xi}(1)\right)\right)_{\xi<\omega_{1}}$ formed by consecutive intervals. The maximality of the intervals implies that $\rho_{\alpha_{\xi}(0)} \neq \rho_{\alpha_{\xi}(1)}$.

Otherwise, when there are only countably many such intervals, let us see that $T$ is in the closure of a countable set of linear combinations of projections on initial blocks. Let $\left.\left(\left[\alpha_{\xi}(0), \alpha_{\xi}(1)\right)\right)\right)_{\xi<\eta}$ be an increasing enumeration of all such intervals where $\eta$ is a countable ordinal. Define for $0<\xi \leq \eta$

$$
T_{\xi}=P_{\sup \left\{\alpha_{\theta}(1): \theta<\xi\right\}} \circ T
$$

noting that $T_{\eta}=T$. 
Now we prove by transfinite induction on $\xi \leq \eta$ that $T_{\xi}$ is in the closure of linear span of operators of the form $\rho_{\alpha_{\xi}(0)}\left[P_{\alpha_{\xi}(1)}-P_{\alpha_{\xi}(0)}\right]$ for $\xi<\eta$. For $\xi<\eta$ we have

$$
T_{\xi+1}=T_{\xi}+\rho_{\alpha_{\xi}(0)}\left[P_{\alpha_{\xi}(1)}-P_{\alpha_{\xi}(0)}\right],
$$

so the successor inductive step is clear. If $\xi$ is a limit ordinal, fix an $x \in B$, note that there is a (countable) strictly increasing sequence $\left(\xi_{n}\right)_{n \in N}$ such whose supremum is $\xi$. The density of $\left\{1_{\{\alpha\}}: \alpha<\lambda\right\}$ implies that there is a finite linear combination $y$ of these vectors for which is close to $x$. Hence

$$
T_{\xi}(x)-T_{\xi_{n}}(x)=\left(T_{\xi}-T_{\xi_{n}+1}\right)(x-y)
$$

holds if $n$ is large enough, so it is small as well since $T_{\xi}$ and $T_{\xi_{n}}$ are uniformly bounded. In other words $T_{\xi_{n}}$ 's converge to $T_{\xi}$ in the strong operator topology and so, the inductive assumption implies that $T_{\xi}$ is in the closure of the linear span of operators of the form $\rho_{\alpha_{\xi}(0)}\left[P_{\alpha_{\xi}(1)}-P_{\alpha_{\xi}(0)}\right]$ for $\xi<\eta$ which completes the proof of the claim. Note that we actually proved that it is the limit of a transfinite sequence of these operators in the sense of [15].

By the above claim we can assume the existence of the pairs as in the claim, moreover we can assume that

$$
\left|\rho_{\alpha_{\xi}(0)}-\rho_{\alpha_{\xi}(1)}\right|>\varepsilon
$$

for some $\varepsilon>0$. Now use D3) to find $X \in\left[\omega_{1}\right]^{k}$ such that $\left\{a_{\xi}: \xi \in X\right\} \in \mathcal{D}$ where $\sqrt{k} \varepsilon \geq(\sqrt{2}+\sqrt{5})\|T\|$. It follows from Claim 4 and AD1) that $\nu\left(1_{\cup D}\right)+\left\|1_{\cup D}\right\|_{\mathcal{A}} \leq \sqrt{2}+\sqrt{5}$, while on the other hand

$$
\nu\left(T\left(1_{\cup D}\right)\right) \geq \Sigma\left\{\left|\rho_{a_{\xi(0)}}-\rho_{a_{\xi(1)}}\right|: \xi \in X\right\}>\sqrt{k} \varepsilon \geq(\sqrt{2}+\sqrt{5})\|T\|
$$

which is a contradiction and which completes the proof of the lemma.

\section{The consistency of the infinitary combinatorics needed}

Theorem 3.1. It is relatively consistent with $Z F C$ that there exists a $W C G$ Banach space of density $\omega_{2}$ which has few operators. For every cardinal $\lambda$ it is relatively consistent with ZFC that there exists a WCG Banach space of density $\lambda$ where all operators are sums of a separable range operator and a diagonal operator with respect to a certain Marcuševič's basis.

This theorem is an immediate consequence of Lemma 2.1 and the following: 
Lemma 3.2. Let $\lambda$ be any cardinal. There is a c.c.c. forcing notion $P$ such that in $V^{P}$ there are families $\mathcal{A}, \mathcal{B}, \mathcal{D}, \mathcal{F}$ satisfying properties $\left.\left.\mathrm{A} 1\right)-\mathrm{DF} 1\right)$ with the exception of D3). It is consistent that there is a c.c.c. forcing notion $P$ such that in $V^{P}$ there are families $\mathcal{A}, \mathcal{B}, \mathcal{D}, \mathcal{F}$ satisfying all properties A1)-DF1) for $\lambda=\omega_{2}$.

Proof. Note that in order to obtain the first part of the lemma we can choose $\mathcal{D}$ to be empty and $\mathcal{F}=[\lambda]^{\omega}$, that is we just need to add families $\mathcal{A}$ and $\mathcal{B}$ which satisfy the properties A1)-AB2). The c.c.c. forcing $P$ required by the theorem consists of the conditions $p$ of the form $\left(a_{p}, \mathcal{A}_{p}, \mathcal{B}_{p}\right)$ where

1) $a_{p} \in[\lambda]<\omega$.

2) $\mathcal{A}_{p} \subseteq \mathcal{P}\left(a_{p}\right), \mathcal{B}_{p} \subseteq \mathcal{P}\left(a_{p}\right)$.

3) $\mathcal{A}_{p}, \mathcal{B}_{p}$ are closed under subsets.

4) $\mathcal{A}_{p} \cap \mathcal{B}_{p} \subseteq\left[a_{p}\right]^{1}$.

The order is defined by $p \leq q$ if and only if $a_{p} \supseteq a_{q}, \mathcal{A}_{p} \supseteq \mathcal{A}_{q}, \mathcal{B}_{p} \supseteq \mathcal{B}_{q}$.

Claim 1. P satisfies the c.c.c.

Proof of the Claim 1. Suppose that $\left(p_{\xi}: \xi<\omega_{1}\right) \subseteq P$. We may w.l.o.g. assume that $\left(a_{p_{\xi}}: \xi<\omega_{1}\right)$ form a $\Delta$-system with the root $\Delta$ such that there are order preserving bijections $\pi_{\xi \eta}: a_{\xi} \rightarrow a_{\eta}$ constant on $\Delta$ which lift up to isomorphisms $\pi_{\xi \eta}^{*}$ of the conditions.

Choose any $\xi<\eta<\omega_{1}$. Let $p_{\xi}=p, p_{\eta}=q$. We may define $r \leq p, q$ as follows

$$
r=\left(a_{p} \cup a_{q}, \mathcal{A}_{p} \cup \mathcal{A}_{q}, \mathcal{B}_{p} \cup \mathcal{B}_{q}\right) .
$$

It is easy to see that the requirements 1)-4) are satisfied, which completes the proof of Claim 1.

Let $G$ be a $P$-generic filter over $V$. We define $X=\bigcup\left\{a_{p}: p \in G\right\}$ $\mathcal{A}=\bigcup\left\{\mathcal{A}_{p}: p \in G\right\} \mathcal{B}=\bigcup\left\{\mathcal{B}_{p}: p \in G\right\}$. An easy density argument gives that $X=\lambda$.

Clearly A1)-AB1) are satisfied for $\mathcal{A}, \mathcal{B}$ and $X$ as above. So to finish the proof of the first part of Lemma 3.2 we need the following:

Claim 2. $\mathcal{A}, \mathcal{B}$ satisfy $\mathrm{AB} 2)$.

Proof of the Claim 2. Let $\left(\dot{b}_{\xi}: \xi<\omega_{1}\right)$ be names for disjoint pairs of elements of $\lambda$ in the generic extension. Let $\left(b_{\xi}: \xi<\omega_{1}\right)$ be pairs of $\lambda$ and $\left(p_{\xi}: \xi<\omega_{1}\right) \subseteq P$ such that $p_{\xi} \Vdash \dot{b}_{\xi}=\check{b}_{\xi}$ and $b_{\xi}=\left\{b_{\xi}(0), b_{\xi}(1)\right\}$ for $\xi<\omega_{1}$. Choose $\Delta$ and $\pi_{\xi \eta}$ as in the proof of Claim 1. By the disjointness of the pairs in the extension and the c.c.c. of $P$ we may w.l.o.g. assume that $b_{\xi} \subseteq a_{\xi}-\Delta$. Fix $k \in \omega$ and consider $\xi_{1}, \ldots, \xi_{k}$, we put

$r=\left(\bigcup_{i \leq k} a_{p_{\xi_{i}}}, \bigcup_{i \leq k} \mathcal{A}_{p_{\xi_{i}}} \cup \mathcal{P}\left(\left\{b_{\xi_{1}}(1), \ldots, b_{\xi_{k}}(1)\right\}\right), \bigcup_{i \leq k} \mathcal{B}_{p_{\xi_{i}}} \cup \mathcal{P}\left(\left\{b_{\xi_{1}}(0), \ldots, b_{\xi_{k}}(0)\right\}\right)\right)$. 
First we need to check that $r \in P$, but the conditions 1)-4) are trivially satisfied. It is easy to see that the condition $r$ forces that

$$
\begin{array}{r}
\left\{b_{\xi_{1}}(1), \ldots, b_{\xi_{k}}(1)\right\} \in \mathcal{A}, \\
\left\{b_{\xi_{1}}(0), \ldots, b_{\xi_{k}}(0)\right\} \in \mathcal{B}
\end{array}
$$

which completes the proof of Claim 2 and the first part of the lemma.

Claim 3. It is consistent that there is a function $F:\left[\omega_{2}\right]^{2} \rightarrow\left[\omega_{2}\right]^{\leq \omega}$ with the following two properties:

P1) Whenever $\left(a_{\xi}: \xi<\omega_{1}\right)$ is a $\Delta$-system of finite subsets of $\omega_{2}$ with root $\Delta \subseteq \omega_{2}$ and $k \in \omega$, then there are $\xi_{1}<\ldots<\xi_{k}<\omega_{1}$ such that

$$
\begin{aligned}
& \forall i, j \leq k, i \neq j \forall \alpha \in a_{\xi_{i}}-\Delta \forall \beta \in a_{\xi_{j}}-\Delta \\
& F(\alpha, \beta) \supseteq \bigcup\left\{a_{\xi_{m}} \cap \min (\alpha, \beta): m<i, j\right\} .
\end{aligned}
$$

P2) For every $\alpha<\omega_{2}$, for every finite $E \subseteq \omega_{2}$ and every countable $Z \subseteq$ $\omega_{2}-\alpha$ there is a finite $E^{\prime} \subseteq \omega_{2}-Z$ such that there is an order preserving bijection $\pi: E \rightarrow E^{\prime}$ which is the identity on $E \cap E^{\prime}$ and satisfies for every $\alpha, \beta \in E$ :

$$
\pi[F(\alpha, \beta) \cap E]=F(\pi(\alpha), \pi(\beta)) \cap E^{\prime} .
$$

Proof of the Claim 3. Function with the $\Delta$-property of [3] has P1). Also using the ideas of [18] one can conclude that if Chang's Conjecture fails, then there is such a function. To obtain P2) we fix the function with the $\Delta$-property of [3] and recall that it is added by a $\sigma$-closed forcing. Thus, one can assume that $E$ and $\alpha$ and $X$ as above are in the ground model of Section 9 of [3]. Given any condition $p$ of the forcing of Section 9 of [3], construct a copy $q$ of $p$ such that there is an increasing order-preserving bijection $\pi: \operatorname{base}(p) \rightarrow \operatorname{base}(q)$ and $\operatorname{base}(p) \cap \alpha=\operatorname{base}(q) \cap \alpha=\operatorname{base}(p) \cap \operatorname{base}(q)$ and $Z \cap(\operatorname{base}(q)-$ base $(p))=\emptyset$. Now amalgamate the two conditions as in $[3]$ to a condition $r$ and note that $r$ forces that $E^{\prime}=\pi[E]$ satisfies $(* *)$.

Let $F:\left[\omega_{2}\right]^{2} \rightarrow\left[\omega_{2}\right] \leq \omega$ be as in Claim 3. We construct a family $\mathcal{F} \subseteq\left[\omega_{2}\right]^{\omega}$ and a c.c.c. forcing $Q$ which adds $\mathcal{A}, \mathcal{B}, \mathcal{D}$ such that all the properties A1)DF1) are satisfied.

The conditions $p$ of the forcing $Q$ are of the form $p=\left(a_{p}, \mathcal{A}_{p}, \mathcal{B}_{p}, \mathcal{D}_{p}\right)$ where $\left(a_{p}, \mathcal{A}_{p}, \mathcal{B}_{p}\right) \in P$ from the beginning of the proof for $\lambda=\omega_{2}$ and additionally we have:

5) Elements of $\mathcal{D}_{p}$ are sets of consecutive pairs of $a_{p}$.

6) $\mathrm{AD} 1)$ and BD1) of Section 2 are satisfied.

7) Whenever $D^{1}, D^{2} \in \mathcal{D}_{p} ; d_{1}^{i}, d_{2}^{i} \in D^{i}, d_{1}^{i}<d_{2}^{i}, \alpha \in d_{1}^{1} \cap d_{1}^{2}, \beta \in d_{2}^{1} \cap d_{2}^{2}$, then

$$
\left\{d \in D^{1}: d<d_{1}^{1}\right\}=\left\{d \in D^{2}: d<d_{1}^{2}\right\},
$$




$$
\bigcup\left\{d \in D^{1}: d<d_{1}^{1}\right\}=\bigcup\left\{d \in D^{2}: d<d_{1}^{2}\right\} \subseteq F(\alpha, \beta) .
$$

(Note that $D^{1}$ may be equal to $D^{2}$ above.)

We need to prove that $Q$ satisfies the c.c.c and adds the required families $\mathcal{A}, \mathcal{B}, \mathcal{D}, \mathcal{F}$. The following claim uses $F$ in a key way.

Claim 4. $Q$ satisfies the c.c.c.

Proof of the Claim 4. Let $\left(p_{\xi}: \xi<\omega_{1}\right) \subseteq Q$. We may w.l.o.g. assume that $\left(a_{p_{\xi}}: \xi<\omega_{1}\right)$ form a $\Delta$-system with the root $\Delta$ such that there are order preserving bijections $\pi_{\xi \eta}: a_{\xi} \rightarrow a_{\eta}$ constant on $\Delta$ which lift up to isomorphisms $\pi_{\xi \eta}^{*}$ of the conditions.

Further thinning out, we may w.l.o.g. assume that $F\left(\delta_{1}, \delta_{2}\right) \cap\left(a_{\xi}-\Delta\right)=\emptyset$ for any $\delta_{1}, \delta_{2} \in \Delta$. Choose any $\xi<\eta<\omega_{1}$. Let $p_{\xi}=p, p_{\eta}=q$. We may define $r \leq p, q$ as follows

$$
r=\left(a_{p} \cup a_{q}, \mathcal{A}_{p} \cup \mathcal{A}_{q}, \mathcal{B}_{p} \cup \mathcal{B}_{q}, \mathcal{D}_{p} \cup \mathcal{D}_{q}\right) .
$$

It is easy to see that the requirements 1)-6) are satisfied. To see 7), let $D^{1}, D^{2} \in \mathcal{D}_{r}$. we may w.l.o.g. assume that $D^{1} \in \mathcal{D}_{p}-\mathcal{D}_{q}$ and $D^{2} \in \mathcal{D}_{q}-\mathcal{D}_{p}$. Thus if $\alpha, \beta$ are like in 7), then they both belong to $\Delta$. By 7) (b) for $p$ and $q$ and by the choice of $p$ and $q$ we have that

$$
\begin{aligned}
& \bigcup\left\{d \in D^{1}: d<d_{1}^{1}\right\} \subseteq \Delta, \\
& \bigcup\left\{d \in D^{2}: d<d_{1}^{2}\right\} \subseteq \Delta,
\end{aligned}
$$

so by the isomorphism of $p$ and $q$ we have 7) (a) and 7) (b), which completes the proof of Claim 4.

Let $G$ be a $P$-generic filter over $V$. We define $X=\bigcup\left\{a_{p}: p \in G\right\}, \mathcal{A}=$ $\bigcup\left\{\mathcal{A}_{p}: p \in G\right\}, \mathcal{B}=\bigcup\left\{\mathcal{B}_{p}: p \in G\right\}, \mathcal{D}=\bigcup\left\{\mathcal{D}_{p}: p \in G\right\}, \mathcal{F}=\{Y \cap X: Y$ is closed under $F\}$ where for a set $Y$ to be closed under $F$ means that that $F(\alpha, \beta) \subseteq Y$ whenever $\alpha, \beta \in Y$. Clearly A1)-AB1), D1), AD1), BD1), F1) are satisfied for $\mathcal{A}, \mathcal{B}, \mathcal{D}, \mathcal{F}$ and $X$ instead of $\lambda=\omega_{2}$. It is an easy density argument to show that $X$ has cardinality $\omega_{2}$, so it is enough to verify all the properties from Section 2 for $X$ instead of $\lambda=\omega_{2}$.

It is also an elementary argument that D2), D4) and DF1) follow from 7): for D2), suppose D2) fails and take the four upper pairs from $D$ out of the five which witness the failure of D2). This implies that at least two distinct pairs of $D^{\prime}$ are involved i.e., that the hypothesis of 7 ) is satisfied, but 7) (a) implies that the fifth lowest pair belongs to both $D$ and $D^{\prime}$ which contradicts the hypothesis of D2) and the consecutiveness of the pairs in $D^{\prime}$.

For D4) let $p \in Q$, and let $\dot{D}$ and $\dot{X}_{1}, \dot{\alpha}$ be $Q$-names for objects mentioned in D4). Using the c.c.c of $Q$ and the decision property of forcing, there are 
$D \in\left[[\lambda]^{2}\right]^{<\omega}, \alpha \in \lambda$ and $Y \subseteq\left[\omega_{2}-\alpha\right]^{\leq \omega}$ such that $D \in \mathcal{D}_{p}$ and

$$
p \| \dot{D}=\check{D}, \dot{X}_{1} \subseteq \check{Y} \cap \dot{X}, \dot{\alpha}=\check{\alpha} .
$$

Using (**) we obtain $E^{\prime}$ as in (**) for $E=a_{p}$ and $Z=Y \cup\left(a_{p}-\alpha\right)$. Let $\pi: a_{p} \rightarrow E^{\prime}$ be the restriction of the bijection from (**). Construct $q \in Q$ as follows

$$
\left.q=\left(a_{q},\left\{\pi[a]: a \in \mathcal{A}_{p}\right\},\left\{\pi[b]: a \in \mathcal{B}_{p}\right\},\left\{\{\pi[d]: d \in D\}: D \in \mathcal{D}_{p}\right\}\right\}\right)
$$

where $a_{q}=E^{\prime}$. Now, $q$ is a condition of $Q$ since $p$ is a condition of $Q$ and by $(* *)$.

It is enough to amalgamate $p$ and $q$ to a condition $r \in Q$. This is done as in Claim 4 since $\pi$ is the required bijection. It is clear that $r$ forces that $D^{\prime}=\pi[D]$ and $D$ satisfy D4), which completes the proof of D4).

For DF1) suppose $Y$ is closed under $F$, i.e., $X \cap Y$ is in $\mathcal{F}$ and let $D$, $d, d^{\prime}, d^{\prime \prime}$ be as in DF1). Let $\alpha \in d^{\prime} \cap X$ and $\beta \in d^{\prime \prime} \cap X$. Now by 7) (b) applied for $D^{2}=D^{1}=D$, we have

$$
d \subseteq F(\alpha, \beta) \subseteq X
$$

which was required. So, to finish the proof of Lemma 3.2 we need to verify AB2) and D3).

Claim 5. $\mathcal{A}, \mathcal{B}$ and $X$ satisfy $\mathrm{AB} 2)$.

Proof of the Claim 5. Let $\left(\dot{b}_{\xi}: \xi<\omega_{1}\right)$ be names for disjoint pairs of elements of $\dot{X}$ in the generic extension. Let $\left(b_{\xi}: \xi<\omega_{1}\right)$ be pairs of $\omega_{2}$ and $\left(p_{\xi}: \xi<\omega_{1}\right) \subseteq Q$ such that $p_{\xi} \Vdash \dot{b}_{\xi}=\check{b}_{\xi}$ and $b_{\xi}=\left\{b_{\xi}(0), b_{\xi}(1)\right\}$ for $\xi<\omega_{1}$. Choose $\Delta$ and $\pi_{\xi \eta}$ as in the proof of Claim 4. By the disjointness of the pairs and the c.c.c. of $Q$ we may w.l.o.g. assume that $b_{\xi} \subseteq a_{p_{\xi}}-\Delta$. Fix $k \in \omega$ and consider $\xi_{1}, \ldots, \xi_{k}$, we put

$$
\begin{aligned}
r= & \left(\bigcup_{i \leq k} a_{p_{\xi_{i}}}, \bigcup_{i \leq k} \mathcal{A}_{p_{\xi_{i}}} \cup \mathcal{P}\left(\left\{b_{\xi_{1}}(1), \ldots, b_{\xi_{k}}(1)\right\}\right),\right. \\
& \left.\bigcup_{i \leq k} \mathcal{B}_{p_{\xi_{i}}} \cup \mathcal{P}\left(\left\{b_{\xi_{1}}(0), \ldots, b_{\xi_{k}}(0)\right\}\right), \bigcup_{i \leq k} \mathcal{D}_{p_{\xi_{i}}}\right) .
\end{aligned}
$$

First we need to check that $r \in P$, but the conditions 1)-5) are trivially satisfied. Also 6$)$ is not difficult to verify as for example both $\left\{b_{\xi_{1}}(0), \ldots, b_{\xi_{k}}(0)\right\}$ and $\left\{b_{\xi_{1}}(1), \ldots, b_{\xi_{k}}(1)\right\}$ can intersect elements of $\mathcal{D}_{r}$ only in one element; and if $a \in \mathcal{A}_{p_{\xi_{i}}}$ and $D \in \mathcal{D}_{p_{\xi_{j}}}$ one can use the isomorphism among the conditions and 6 ) for one of the conditions $p_{\xi_{i}}$ or $p_{\xi_{j}}$. The verification of 7) is as in Claim 4.

It is easy to see that the condition $r$ forces that

$$
\left\{b_{\xi_{1}}(1), \ldots, b_{\xi_{k}}(1)\right\} \in \mathcal{A},
$$




$$
\left\{b_{\xi_{1}}(0), \ldots, b_{\xi_{k}}(0)\right\} \in \mathcal{B}
$$

which completes the proof of Claim 5.

The following claim uses $F$ in a key way.

Claim 6. $\mathcal{D}$ and $X$ satisfy D3).

Proof of the Claim 6. Let $k \in \omega$ and let $\left(\dot{d}_{\xi}: \xi<\omega_{1}\right)$ be names for consecutive (and in particular disjoint) pairs of elements of $\dot{X}$ in the generic extension. Let $\left(d_{\xi}: \xi<\omega_{1}\right)$ be pairs of $\omega_{2}$ and $\left(p_{\xi}: \xi<\omega_{1}\right) \subseteq Q$ such that $p_{\xi} \|-\dot{d}_{\xi}=\check{d}_{\xi}$. Choose $\Delta$ and $\pi_{\xi \eta}$ as in the proof of Claim 4 . By the consecutiveness of the pairs and the c.c.c. of $Q$ we may w.l.o.g. assume that $d_{\xi} \subseteq a_{p_{\xi}}-\Delta$ and $\left(d_{\xi}: \xi<\omega_{1}\right)$ are consecutive pairs (just take amalgamations as in Claim 5 to get the consecutiveness). Fix $k \in \omega$ and use the property of $F$ to obtain $\xi_{1}, \ldots, \xi_{k}$ satisfying Claim 3 for $\left(a_{p_{\xi}}: \xi<\omega_{1}\right)$ as above; we put

$$
r=\left(\bigcup_{i \leq k} a_{p_{\xi_{i}}}, \bigcup_{i \leq k} \mathcal{A}_{p_{\xi_{i}}}, \bigcup_{i \leq k} \mathcal{B}_{p_{\xi_{i}}}, \bigcup_{i \leq k} \mathcal{D}_{p_{\xi_{i}}} \cup\left\{\left\{d_{\xi_{i}}: i \leq k\right\}\right\}\right) .
$$

First we need to check that $r \in P$, but the conditions 1)-5) are trivially satisfied. Also 6) is not difficult to verify as for example $\left\{d_{\xi_{i}}: i \leq k\right\}$ can intersect elements of $\mathcal{A}_{r}$ only in one pair; and if $a \in \mathcal{A}_{p_{\xi_{i}}}$ and $D \in \mathcal{D}_{p_{\xi_{j}}}$ one can use the isomorphism among the conditions and 6) for one of the conditions $p_{\xi_{i}}$ or $p_{\xi_{j}}$. Now let us verify 7). If $D^{1}, D^{2} \in \bigcup_{i \leq k} \mathcal{D}_{p_{\xi_{i}}}$ we may use the same argument as in the proof of Claim 4. Now assume that $D^{1}=\left\{\left\{d_{\xi_{i}}: i \leq k\right\}\right\}$ and $D^{2} \in \mathcal{D}_{p_{\xi_{i}}}$ for some $1 \leq i \leq k$. But this implies that the hypothesis of 7) fails.

Finally let $D^{1}=D^{2}=\left\{\left\{d_{\xi_{i}}: i \leq k\right\}\right\}$. We need to verify 7 ) (b), but this follows from the property of $F$ in Claim 3 .

It is also immediate that $r$ forces that $\left\{\left\{\dot{d}_{\xi_{i}}: 1 \leq i \leq k\right\}\right\} \in \mathcal{D}$. This completes the proof of the lemma.

Remark. S. Todorčević ([19]) has shown that the existence of the above families follows from the combinatorial principle $\square \omega_{1}$ via his $\rho$-function. It follows that in particular one can have such a Banach space of density bigger than $2^{\omega}$.

\section{Projections from Chang's conjecture}

Recall that if $M$ together with some relations (including constants and functions) is a structure for some first-order language, then $N$ is an elementary substructure of $M$ whenever for every $x_{1}, \ldots, x_{n} \in N$ the formula 
$\phi\left(x_{1}, \ldots x_{2}\right)$ of the language is true in $N$ if and only if it is true in $M$. Elementary submodels provide a quick way of obtaining substructures closed under all finitary operations we consider. The reader may consult Section 1 of [6] for some more information. Chang's conjecture (see [14], [8]) is the statement which says that every structure of the form $\left(M ; \omega_{1}, R_{1}, \ldots, R_{k}\right)$ for $M$ of cardinality $\omega_{2}$ has an elementary substructure with the universe $N$ which in uncountable and such that $N \cap \omega_{1}$ is countable. It is known that it is independent of the axioms of ZFC (assuming the existence of some large cardinals, see [14]). We will consider elementary substructures of fragments $H(\theta)$ of the set-theoretic universe, for $\theta$ sufficiently large to contain all relevant objects. The Löwenheim-Skolem-Tarski theorem provides elementary submodels of such structures of size $\omega_{2}$, and so Chang's conjecture can be applied.

Recall (see e.g. [12]) that a Marcuševič's basis $\left(\left(x_{\alpha}, f_{\alpha}\right): \alpha<\lambda\right)$ of a Banach space $X$ is 1-countably norming if and only if

$$
\|x\|=\sup \left\{|f(x)|:\left\{\alpha: f\left(x_{\alpha}\right) \neq 0\right\} \text { is countable, }\|f\| \leq 1, f \in X^{*}\right\} .
$$

It is noted in [12] (pp. 368-369) that any Marcuševič's basis in a WCG Banach spaces is countably 1-norming.

Lemma 4.1. Suppose that $X$ is a weakly compactly generated space and $\mathcal{B}=\left(\left(x_{\alpha}, f_{\alpha}\right): \alpha<\lambda\right)$ is its Marcuševič's basis. Suppose that $M$ is an elementary substructure of

$$
\left(H(\theta) ; \in, \omega_{1}, \lambda, X,\|\|_{X}, \mathcal{B}\right) .
$$

Then there is a norm-one projection $P: X \rightarrow X$ such that $P\left(x_{\alpha}\right)=x_{\alpha}$ for $\alpha \in M \cap \lambda$ and $P\left(x_{\alpha}\right)=0$ for $\alpha \in \lambda-M$.

Proof. First we will show that the sum

i) $\left[\left\{x_{\alpha}: \alpha \in M \cap \lambda\right\}\right] \oplus\left[\left\{x: f(x)=0\right.\right.$ for all $\left.\left.f \in M \cap X^{*},\|f\| \leq 1\right\}\right]$, is closed and the projection on the first factor is of norm one. We will follow Lemmas VI 2.3 and VI 2.4 of [4] which imply the existence of the desired projections. First we will check the following assertion which correspond to (iii) of VI 2.3 from [4]:

ii) For all $x \in\left[\left\{x_{\alpha}: \alpha \in M \cap \lambda\right\}\right]$ we have

$$
\|x\|=\sup \left\{f(x):\|f\| \leq 1, \quad f \in M \cap X^{*}\right\} .
$$

This can be obtained by the elementarity of $M$. If $x \in\left[\left\{x_{\alpha}: \alpha \in M \cap \lambda\right\}\right]$, there is a finite linear combination with rational coefficients $\sum q_{i} x_{\alpha_{i}}$ with $\alpha_{i} \in M \cap \lambda$ such that $\left\|x-\sum q_{i} x_{\alpha_{i}}\right\|$ is small. Note that $\sum q_{i} x_{\alpha_{i}}$ belongs to $M$, and so, by the elementarity there is a norm-one functional $f$ in $M$ such that $f\left(\sum q_{i} x_{\alpha_{i}}\right)$ is close to the norm of $\sum q_{i} x_{\alpha_{i}}$, which completes the proof of ii). 
So by ii) if $x \in\left[\left\{x_{\alpha}: \alpha \in M \cap \lambda\right\}\right]$ and $\varepsilon>0$, then there is an $f \in M \cap X^{*}$ with $\|f\| \leq 1$ such that $f(x)>\|x\|-\varepsilon$, for such an $f$ we have $f(y)=0$ for every $y$ in the second factor of our direct sum i), so

$$
\|x\|-\varepsilon<f(x)=f(x+y) \leq\|x+y\|,
$$

which implies that $\|x\| \leq\|x+y\|$. As the interesection of the factors is $\{0\}$ a standard argument allows us to conclude that the projection of the first factor is well-defined and is of norm one and so the sum i) is closed.

To conclude that the sum i) is the entire space $X$, we essentially follow [12]. Whenever $f$ is a functional in $M$, the countable (since the basis is 1-countably norming) set $\left\{\alpha: f\left(x_{\alpha}\right) \neq 0\right\}$ belongs to $M$ and so, is included in $M$ (see $[6,1.6]$ ), i.e., $x_{\beta}$ is in the second factor if $\beta \in \lambda-M$ and the span of the vectors of any Marcuševič basis is norm dense in $X$. It also follows that $P\left(x_{\beta}\right)=0$ for $\beta \in \lambda-M$.

Theorem 4.2. Assume Chang's Conjecture. Suppose that $X$ is a WCG Banach space of density $\omega_{2}$ and $\left(P_{\alpha}: \omega \leq \alpha \leq \omega_{2}\right)$ is a projectional resolution of identity on $X$. There is a norm-one projection on $X$ which is not of the form $T+S$, where $T$ is in the closure (in the strong operator topology) of the linear span of a countable set of $P_{\alpha}$ 's and $S$ has separable range. In particular $X$ has more than few operators.

Proof. It is known that a WCG space has a Marcuševič's basis (see [13]), so let $\left(x_{\alpha}, f_{\alpha}: \alpha<\lambda\right)$ be such a basis.

Let $M$ be an elementary substructure of

$$
\left(H(\theta) ; \in, \omega_{1}, \lambda, X,\|\|_{X}, \mathcal{B}\right)
$$

obtained from Chang's Conjecture. Let $P: X \rightarrow X$ be as in the previous lemma. Let us suppose that it is of the form $T+S$ as in the theorem and let us arrive at a contradiction.

As there is a countable ordinal $\alpha$ which does not belong to $M$, by the elementarity, for any $\beta \in M \cap \omega_{2}$ we have that $\beta+\alpha \notin M$. On the other hand $M \cap \omega_{2}$ is uncountable. Thus it is easy to obtain a sequence $\left(\left(\beta_{\xi}, \alpha_{\xi}\right): \xi<\omega_{1}\right)$ of consecutive pairs of ordinals in $\omega_{2}$ such that $\beta_{\xi}<\alpha_{\xi}$ and $\beta_{\xi} \in M$ while $\alpha_{\xi} \notin M$ (e.g. put $\alpha_{\xi}=\beta_{\xi}+\alpha$ where $\alpha$ as above and thin the sequence out if necessary). We may w.l.o.g. assume that all the vectors $x_{\beta_{\xi}}, x_{\alpha_{\xi}}$ for $\xi<\omega_{1}$ are not in the range of $S$. Let $A \subseteq \omega_{2}$ be the countable set of all ordinals $\alpha$ in $\omega_{2}$ such that $P_{\alpha}$ appears in the finite linear combinations which have $T$ in the closure. Clearly there is a $\xi<\omega_{1}$ such that for every $\alpha \in A$ we have

$$
\alpha<\beta_{\xi} \quad \Leftrightarrow \quad \alpha<\alpha_{\xi} .
$$


This means that $T\left(x_{\beta_{\xi}}\right)=T\left(x_{\alpha_{\xi}}\right)$. However $P\left(x_{\beta_{\xi}}\right)=x_{\beta_{\xi}}$ and $P\left(x_{\alpha_{\xi}}\right)=0$, i.e.,

$$
\begin{gathered}
T\left(x_{\beta_{\xi}}\right)+S\left(x_{\beta_{\xi}}\right)=x_{\beta_{\xi}}, \\
T\left(x_{\alpha_{\xi}}\right)+S\left(x_{\alpha_{\xi}}\right)=0 .
\end{gathered}
$$

Subtracting these equations, we obtain

$$
S\left(x_{\beta_{\xi}}\right)-S\left(x_{\alpha_{\xi}}\right)=S\left(x_{\beta_{\xi}}-x_{\alpha_{\xi}}\right)=x_{\beta_{\xi}},
$$

which contradicts the fact that $x_{\beta_{\xi}}$ is not in the range of $S$ and completes the proof of the theorem.

Theorem 4.3. Suppose that $X$ is a WCG Banach space of density $\lambda>\omega_{2}$ and $\left(P_{\alpha}: \omega \leq \alpha \leq \lambda\right)$ is a projectional resolution of identity on $X$. There is a norm-one projection on $X$ which is not of the form $T+S$, where $T$ is in closure (in the strong operator topology) of the linear span of countably many operators $\left(P_{\alpha}: \omega \leq \alpha \leq \lambda\right)$ and $S$ has separable range. In particular $X$ has more than few operators.

Proof. We repeat the previous proof noting that the classical LöwenheimSkolem-Tarski theorem provides the desired elementary submodel. Indeed, any elementary submodel $M$ of a sufficiently large fragment of set-theory which contains $\omega_{2}$, is of cardinality $\omega_{1}$ and such that the cofinality of $M \cap \lambda$ is uncountable provides the desired sequence $\left(\left(\alpha_{\xi}, \beta_{\xi}\right): \xi<\omega_{1}\right)$ as in the previous proof. Just take $\beta \in \omega_{2}-M$ and construct by induction a sequence $\left(\alpha_{\xi}\right)_{\xi<\omega_{1}}$ such that

$$
\alpha_{\xi}+\beta=\beta_{\xi}<\alpha_{\xi}+\omega_{2} \leq \alpha_{\xi+1}
$$

Theorem 4.4. Suppose that $X$ is a WCG Banach space of density bigger than $2^{\omega_{1}}$. Then for every Marcuševič's basis of $X$ there is an operator on $X$ which is not of the form a separable range operator plus a diagonal operator with respect to the basis.

Proof. This is just a counting argument. Using the standard way of constructing projections in WCG spaces (see [5]) one can obtain $\left(2^{\omega_{1}}\right)^{+}$distinct projections $P_{A}$ on blocks $A \subseteq \lambda$ of cardinality $\omega_{1}$. One can assume that $A$ 's form a $\Delta$-system whose root is included in some $B$ of cardinality $\omega_{1}$ on which there is a projection. By subtracting $P_{B}$ from $P_{A}$ 's we may assume that $A$ 's are pairwise disjoint. Now, there are at most $2^{\omega_{1}}$ approximate behaviours of the norm on finite rational linear combinations of vectors from a dense set in a Banach space of density $\omega_{1}$, i.e., we obtain that the ranges 
of two projections $P_{A}$ and $P_{A^{\prime}}$ are isomorphic. So consider $T \circ P_{A}$, where $T$ is the isomorphism. It is clear that it is not a diagonal plus a separable range operator.

Remark. Note that Theorem 3.1 provides the consistency of the existence of WCG spaces of density $\lambda$ where every operators is the sum of a diagonal operator plus a separable range operator for each cardinal $\lambda$ in the usual sense that the value of this cardinal on the exponential scale is unspecified. Actually our proof of 3.1 gives $\lambda \leq 2^{\omega}$. Theorem 4.1 shows that it cannot be improved above $2^{\omega_{1}}$, however the value of $\lambda$ on the cardinal scale can be arbitrarily large, i.e., e.g., we can have $\lambda=\omega_{5}, \omega_{\omega_{1}}$ etc.

Remark. Note that the above results imply that the quantity of projections in WCG spaces depends on the quantity of elementary submodels. It would be interesting to construct (without any aditional assumptions) WCG Banach spaces of large densities where all projections are induced by elementary submodels (of various cardinalities) obtaining an unconditional version of Warks's modification of Shelah's and Steprāns' space. This would be probably the right notion of "few projections" in a WCG space. Also one could try to control separable range operators (which are completely arbitrary in our construction) as in [2].

\section{References}

[1] Amir, D., Lindenstrauss, J., The structure of weakly compact sets in Banach spaces, Ann. of Math. (2) 88 (1968), 35-46.

[2] Argyros, S. A., Lopez-Abad, J., Todorčević, S., A class of Banach spaces with few non strictly singular operators, J. Funct. Analysis 222(2) (2005), 306-384.

[3] Baumgartner, J., Shelah, S., Remarks on superatomic Boolean algebras, Ann. Pure Appl. Logic 33 (1987), 109-129.

[4] Deville, R., Godefroy, G., Zizler, V., Smoothness and Renormings in Banach Spaces, Longman Scientific \& Technical, Harlow, 1993.

[5] Diestel, J., Geometry of Banach Spaces - Selected Topics, Lecture Notes in Math. 485, Springer, New York, 1975.

[6] Dow, A., Applications of elementary submodels to topology, Topology Proc. 12(1) (1988), 17-73.

[7] Dunford, N., Schwartz, J., Linear Operators; Part I. General Theory, Interscience Publishers, Inc., New York, Fourth printing, 1967.

[8] Kanamori, A., The Higher Infinite. Large Cardinals in Set Theory from Their Beginnings, Springer-Verlag, Berlin, 1994.

[9] Koszmider, P., On the existence of strong chains in $\mathcal{P}\left(\omega_{1}\right) /$ Fin, J. Symbolic Logic 63(3) (1998), 1055-1062.

[10] Koszmider, P., On strong chains of uncountable functions, Israel J. Math. 118 (2000), $289-315$. 
[11] Kunen, K., Set Theory; An Introduction to Independence Proofs, Stud. Logic Found. Math. 102, North Holland Publishing Co., Amsterdam, 1980.

[12] Pličko, A. On projective resolutions of the identity operator and Marcuševič's bases, Dokl. Akad. Nauk SSRR 25(2) (1982), 386-389.

[13] Reif, J., A note on Marcuševič's bases in weakly compactly generated Banach spaces, Comm. Math. Univ. Carolin. 15(2) (1974), 335-340.

[14] Rowbottom, F., Some strong axioms of infinity incompatible with the axiom of constructibility, Ann. Math. Logic 3 (1971), 1-44.

[15] Singer, I., Bases in Banach Spaces II, Springer-Verlag, Berlin- New York, 1981.

[16] Shelah, S., A Banach space with few operators, Israel J. Math. 30 (1978), 181-191.

[17] Shelah, S., Steprāns, J., A Banach space on which there are few operators, Proc. Amer. Math. Soc. 104 (1988), 101-105.

[18] Todorčević, S., Remarks on Martin's axiom and the continuum hypothesis, Canad. J. Math. 43 (1991), 832-851.

[19] Todorčević, S., Coherent sequences, in "Handbook of Set-Theory", (to appear).

[20] Velleman, D., Simplified morasses, J. Symbolic Logic 49(1) (1984), 257-271.

[21] Wark, H., A nonseparable reflexive Banach space on which there are few operators, J. London Math. Soc. (2) 64(3) (2001), 675-684.

[22] Zapletal, J., Strongly almost disjoint functions, Israel J. Math. 97 (1997), 101-111.

Piotr KosZMider

Departamento de Matemática

UNIVERSIDAdE DE SÃo PAUlo

Caixa Postal: 66281

SÃo Paulo, SP

CEP: 05315-970, BRASIL

E-MAIL: PIOTR@IME.USP.BR

URL: http://www.ime.usp.br/ piotr 